\title{
LAS ACTIVIDADES ECONÓMICAS GLOBALES Y EL ESPACIO URBANO: TRANSFORMACIONES SOCIOTERRITORIALES EN TORNO A LA LOCALIZACIÓN DE CALL CENTERS EN ARGENTINA
}

\author{
Eric Moench \\ Estudiante de la Maestría en Ciencias Sociales del Trabajo \\ Universidad de Buenos Aires, Bs. As. (Argentina) \\ Correo electrónico: moench.eric@gmail.com
}

\begin{abstract}
Recibido: 20 de mayo de 2011. Devuelto para revisión: 22 de mayo de 2011.
Aceptado: 1 de junio de 2011
\end{abstract}

\section{RESUMEN}

La devaluación del tipo de cambio y baja del costo laboral ha masificado el negocio de los call centers en Argentina y ha provocado transformaciones en la configuración de espacios urbanos. Si bien existen empresas de capitales nacionales, quienes lideran el proceso de inversiones son empresas transnacionales: interviene así una lógica de superponer territorios de diferentes escalas (locales, nacionales, globales). La llegada de esta actividad económica -inédita hasta hace pocos años en la mayoría de los espacios locales- ha generado una rejerarquización territorial de las cadenas de valor, producto de la posibilidad de fragmentar la cadena de producción y exportar las tareas de menor valor agregado. Asimismo las empresas reorganizan el territorio a partir de su interacción con actores económicos y sociales locales, estados nacionales y provinciales, en la relación con clientes y proveedores y con la demanda de mano de obra e infraestructura. El presente trabajo, a través de un recorrido por las características de la localización de este servicio, intenta visibilizar adecuadamente estos fenómenos.

Estos cambios son analizados a través de la revisión de formulaciones teóricas ancladas en perspectivas de sociología urbana y sociología de la globalización, que permiten un horizonte más amplio del análisis en cuanto a las transformaciones en los tipos de sociedad que se configuran, así como permiten explorar potencialidades y límites de modelos de desarrollo en espacios urbanos actualmente subordinados.

Palabras claves: Call centers, Actores locales, Empresas transnacionales, Espacios urbanos subordinados

\begin{abstract}
The devaluation of the exchange rate and low labor costs have crowded the business of call centers in Argentina and caused changes in the configuration of urban spaces. Although there are domestic capital companies, who lead the investment process are transnational corporations: a logic intervenes overlap territories of different scales (local, national, global). The arrival of this economic activity, non-existent until recently in most local areas has generated a new territorial hierarchy value chain, due to the possibility of breaking the chain of production and export lower-value tasks. Also firms
\end{abstract}


reorganize the area from their interaction with local social and economic actors, with national and provincial states, the relationship with customers and suppliers and the demand for labor and infrastructure. This paper, through a tour of the features of the location of this service, an attempt to bring these phenomena properly.

These changes are analyzed through a review of theoretical formulations perspectives anchored in urban sociology and sociology of globalization, which allow a wider horizon of the analysis on the changes in the types of companies that are configured as well as for exploring potential and limitations of development models in urban areas currently subordinates.

Keywords: Call centers, local actors, Transnational companies, Subordinate urban spaces

\section{INTRODUCCIÓN}

En Argentina el año 2002 marca el inicio de una serie de transformaciones territoriales producto de nuevas configuraciones económicas, sociales y políticas. Luego de la devaluación del tipo de cambio y baja del costo laboral el sector productivo se reordena. Los actividades económicas de servicios vinculados a las tecnologías de la información y la comunicación $(\mathrm{TICs})^{1}$ se han visto favorecidos en términos de su posicionamiento interno: la relocalización espacial como estrategia clave para disminuir costos aplicada por gran parte de las empresas en países centrales, sumado a la posibilidad de disociación del proceso productivo, reorienta los flujos de inversión extranjera directa que son los que dan impulso a la actividad de call centres $(\mathrm{CCs})^{2}$ en Argentina.

En este artículo presentaremos información sobre las características de esta actividad, y la expansión territorial de CCs tercerizadores ${ }^{3}$ en Argentina, lo que implica la llegada de nuevas actividades económicas inéditas hasta hace pocos años en el país. En su mayoría, se trata de inversiones que se orientan a desarrollar actividades de baja jerarquización, en donde la competitividad se obtiene vía la baja de los costos laborales y el otorgamiento de ventajas impositivas y subsidios estatales hacia las empresas.

La acelerada instalación de CCs en nuestras fronteras -que juzgamos innegable a partir de la evidencia que a continuación mostraremos-, muestra una creciente dinámica global en esta actividad. Si bien existen empresas de capitales nacionales, quienes lideran el proceso de expansión de inversiones son las empresas transnacionales, que generan la

\footnotetext{
1 Las TICs comprenden a los CCs, ya que, entre otras, incluyen actividades de aplicación, almacenamiento y transmisión de la información mediante tecnologías informáticas, electrónicas y comunicacionales.

2 Definimos a los CC como el soporte desarrollado por las empresas para satisfacer determinados aspectos de la relación con sus clientes en base a información a través de un canal comunicacional con soporte informático.

3 Los CCs tercerizadores son los que venden servicios subcontratados a otras empresas. Los seleccionamos para el análisis, pues es este segmento de CCs el que motoriza especialmente un tipo de expansión territorial en el interior del país y el que más empleo genera. La evidencia empírica señala también -no sólo aquí, sino en todo el mundo- que hay peores condiciones laborales en los CC tercerizados frente a los in-house, es decir, los operados por la propia compañía. Véase de Miranda Oliveira Junior (2005).
} 
ampliación de mercados de cobertura que se entrecruzan (locales-nacionales-globales), una rejerarquización de las cadenas globales de valor, nuevos conjuntos de flujos y redes espaciales (mano de obra, empresas, mercancías, información) que se tejen en torno a la actividad.

Estos cambios son observados y analizados a través de la revisión de formulaciones teóricas ancladas en perspectivas de sociología urbana y sociología de la globalización, que permiten un horizonte más amplio del análisis en cuanto a las transformaciones en los tipos de sociedad que se están configurando, así como permiten esbozar algunas conclusiones sobre modelos de desarrollo posibles que se ponen en juego en torno a la localización de CCs en espacios locales, en el marco de las dinámicas de la globalización económica ${ }^{4}$.

\section{ALGUNAS NOTAS SOBRE LA SOCIOLOGÍA URBANA}

La complejidad que abordamos en nuestro objeto de estudio está atravesada por la intervención de actores locales, nacionales y transnacionales involucrados en torno a la expansión de la actividad y por las características productivas y tecnológicas de nuevas actividades en servicios. Estos atributos posibilitan nuevas condiciones de interacción de los actores político-sociales y agentes económicos locales y globales, junto a la utilización de nuevas tecnologías digitales; interviene así una lógica de "apilar" territorios 5 que mutan "estirando" las fronteras de la actividad, tanto físicas como virtuales. Abordamos el análisis del territorio no sólo como una estructura geográfica, sino en un sentido amplio y dinámico, lo que:

“...resulta de la apropiación y valorización del espacio y éstas pueden ser: de carácter instrumental-funcional o de carácter simbólico-expresivo. En un caso lo que predomina es la explotación económica o geopolítica y en el otro lo que se acumula es una sedimentación simbólico-cultural (...) Esta definición de territorio se hace plural cuando se analiza en diferentes escalas y niveles históricamente constituidos y sedimentados que van desde lo local hasta lo supranacional pasando por toda la gama intermedia como es el municipio, la región, la provincia y la nación" (Panaia, 2004, pp. 230-231).

Aquí analizamos este carácter instrumental-funcional del territorio local que, en la complejidad de las nuevas actividades económicas objeto de nuestro estudio, puede asumir diferentes y variadas formas de mutación.

En este sentido interesa presentar brevemente algunas de las posiciones teóricas que han renovado los enfoques tradicionales de la sociología urbana y de la sociología de la

\footnotetext{
4 En la investigación empleamos fuentes de información primarias y secundarias. Se entrevistaron empleados calificados de $\mathrm{CC}$, funcionarios públicos y operadores telefónicos; mientras que las fuentes secundarias fueron recolectadas a partir de bibliografía académica, relevamiento exhaustivo de estadísticas, informes y entrevistas en publicaciones de cámaras empresariales, consultoras y revistas especializadas, revisión de los diarios de sesiones de las legislaturas provinciales y decretos-leyes establecidos en cada provincia y diarios provinciales con noticias pertinentes a nuestro estudio.

$5 \mathrm{El}$ concepto refiere a superponer territorios pertenecientes a diferentes escalas (locales, provinciales, nacionales, globales). Véase Panaia (2004).
} 
globalización, en tanto vuelven a dar relevancia al espacio local, y asimismo lo resignifican a partir de su imbricación profunda con lo social e institucional en la conformación de ese mismo espacio. Se trata de miradas que permiten inscribir el fenómeno de llegada de los CCs a Argentina dentro de un horizonte de transformaciones socioeconómicas de escala mundial y que retoman el problema del poder en la estructuración de los espacios.

El proceso de globalización económica de las dos últimas décadas transforma y (re)organiza la producción a escala global, lo que implica también un reordenamiento territorial de las actividades y de los lugares donde se crea el valor las cosas. En este sentido S. Sassen (2010, pp. 433-434) sostiene que:

“...cuanto más se globalizan y digitalizan las operaciones de los mercados y las empresas, más estratégicas se vuelven las funciones de coordinación y gestión centralizada (...) Es precisamente gracias a la digitalización que se puede lograr la dispersión geográfica de las actividades económicas (ya sea en fabricas, oficinas o puntos de venta de servicios) junto con la simultanea integración organizacional de estas actividades, combinación que aumenta la importancia de las funciones centrales. En este sentido, las ciudades globales, constituyen espacios estratégicos por su combinación de recursos necesarios para dichas funciones centrales"

Este proceso se introduce de forma inevitable en los territorios locales, pues aunque las actividades se realicen fronteras adentro, el capital, la organización y gestión y los mercados de cobertura están organizados a escala global, bien de forma directa, bien mediante una red de vínculos entre los agentes económicos. La posibilidad de deslocalizar tareas y la búsqueda de espacios alternativos en los que conseguir mano de obra barata es un ejemplo de la escala global a la que hacemos referencia y da cuenta de nuevos patrones de localización que han posibilitado la instalación de nuevas actividades económicas, quizás no acordes a la historia productiva de una localidad.

En el caso particular aquí presentado, donde la información se ha convertido en un elemento de producción y dada la posibilidad de su digitalización, se requiere de una transformación de la concepción del espacio en el que los conceptos de proximidad y lejanía, los de centralidad y los de densidad, adquieren nuevos significados: autores como Manuel Castells, o Saskia Sassen han elaborado conceptos que buscan dar cuenta de un espacio que integra estas transformaciones y que obligan a reveer relaciones de poder entre países y a su interior.

En el caso de Castells su concepto de espacio de los flujos da paso a nuevos sistemas jerárquicos de poder que se materializan en redes que estructuran este espacio de los flujos y que representan conceptualmente un determinado tipo de organización material de las prácticas sociales caracterizado como:

“...secuencias de intercambio e interacción determinadas, repetitivas y programables entre las posiciones físicamente inconexas que mantienen los actores sociales en las estructuras económicas, políticas y simbólicas de la sociedad" (Castells, 2002, p. 445) 
Dichos flujos tienen un soporte material electrónico (microelectrónica, telecomunicaciones, procesamiento informático, etc.) que interconectan en red a los actores. El nuevo espacio se compone con esta red de infraestructura tecnológica. Aunque esta infraestructura tecnológica puede estar presente en territorios diversos, pueden coexistir $-\mathrm{y}$ de hecho lo hacen- con notorias desigualdades entre ellos. Castells pone nombres a esta desigualdad territorial: nodos y ejes. Su diferencia radica en que:

“...algunos lugares son intercambiadores, ejes de comunicación que desempeñan un papel de coordinación para que haya una interacción uniforme de todos los elementos integrados en la red. Otros lugares son los nodos de la red, es decir, la ubicación de funciones estratégicamente importantes que constituyen una serie de actividades" (Castells, 2002, p. 446).

Es decir, existen jerarquías territoriales según el peso relativo que tenga la actividad -realizada localmente- en el conjunto de la red. Sin embargo, que las desigualdades territoriales continúen no significa que no ha habido transformaciones en los espacios: en primer lugar se observa que las ciudades que se suman -como nodos o ejes- a este espacio de flujos implica que se conectan en una red global en virtud de los flujos de información, mientras que a la vez restan importancia a la conexión con sus entornos territoriales, o al espacio de los lugares (Castells, 2002).

Es interesante observar estas transformaciones de los lugares recurriendo a la categoría sasseniana de desensamblaje parcial de lo nacional. La autora afirma que:

"La globalización consta en gran parte de una variedad enorme de microprocesos que comienzan a desnacionalizar aquello que se ha construido históricamente como nacional, ya sean las políticas de gobierno, los capitales, las subjetividades políticas, los espacios urbanos, las estructuras temporales" (Sassen, 2010, p. 20).

Nos interesa esta perspectiva, en tanto permite subrayar que cada territorio -con su específica densidad de instituciones y actores- tiene algo que aportar a la dinámica de la globalización y a modelar la apropiación y valorización del espacio en tanto procesos construidos socialmente. Así, se aleja de las concepciones de "suma cero" donde si lo global gana, lo local pierde; es en la intersección de lo local/global donde diría la autora que se hace la nueva era global (Sassen, 2010). Ello lleva inexorablemente a entender ciertos procesos de reformas locales como parte de este proceso: en efecto, la inserción de actividades globales en fronteras locales requiere que se ponga en marcha una serie combinada y específica de leyes, reglamentos, alianzas políticas y acuerdos extraoficiales (Sassen, 2010). Por tanto, los estados necesariamente participan de este proceso. Retomando la actividad de CCs que aquí analizamos, es indudable que el estado ha tenido responsabilidades centrales en la instalación su instalación en Argentina, como más adelante detallaremos.

\section{EL SECTOR SERVICIOS EN ESPACIOS URBANOS}

El sector servicios crece a nivel mundial en cuanto a empleabilidad y a participación en el producto bruto. Sin embargo debemos realizar algunas aclaraciones sobre su 
composición, para no oscurecer los debates académicos posteriores sobre el tipo de sociedades que configuran.

En primer lugar, en el sector servicios se suele agrupar en las estadísticas de los países una serie de actividades con poco de común entre ellas ${ }^{6}$. Junto S. Lash y J. Urry (1994) podemos decir que el sector servicios en sí es un agregado con actividades de carácter ampliamente heterogéneo a su interior; incluso en las denominadas economías de la información, hay servicios al consumidor de escaso valor agregado; en palabras de los autores:

"Es un error considerar los servicios como si fueran homogéneos. La paradoja del empleo en los servicios se puede ilustrar en las actividades que más crecieron en servicios en los últimos años: la banca y las finanzas, que emplean una alta proporción de profesionales, frente al ocio y el turismo que emplean una alta proporción de empleos inferiores. El cambio tecnológico, en servicios, genera una polarización entre el personal hibrido de profesionales y gerentes frente los empleados de nivel bajo" (Lash y Urry, 1994, p. 296).

Esta heterogeneidad en el sector servicios (que más adelante veremos que se replica al interior de los CCs) debe ser explicitada, pues los análisis agregados no permiten sacar conclusiones sobre si su avance implica un mayor desarrollo en los territorios. En este sentido, algunos autores han acuñado el concepto de sociedades posindustriales para dar cuenta de un cambio en la hegemonía de los sectores productivos, donde lo industrial pierde peso frente al sector denominado terciario, es decir, la mayor proporción de la actividad económica y de mano de obra empleada en los territorios pasaría de la producción de bienes a la realización de servicios. El siguiente -y controvertido- paso en esta línea es predecir que en la nueva economía las ocupaciones ejecutivas, profesionales y técnicas crecerían más de prisa que todas las demás y constituirían el núcleo de la nueva estructura social ${ }^{7}$.

Si bien se puede constatar un avance en los niveles de empleabilidad del sector servicios -aunque ello no implica un retroceso de lo fabril, de hecho, el auge de muchos servicios está directamente ligado con la fabricación- nos interesa discutir con esta concepción teórica que ha relacionado estos cambios productivos con sociedades más igualitarias o desarrolladas: en su perspectiva las actividades que incluyen componentes tecnológicos para su desarrollo (y ellas se encontraban principalmente en el sector servicios) producen un salto de desarrollo para los países y la mano de obra que se desempeñe en estos sectores. En este sentido, el presente trabajo también puede ser leído en clave de respuesta a la teoría posindustrialista, pues la realidad se ha ocupado de desmentir este cuadro por ellos predicho, al menos para territorios que no son nodos en el engranaje de la sociedad red. Más adelante presentaremos evidencia empírica precisa del avance territorial y generación de empleo en los CCs, que muestra cómo el avance de estas actividades en el sector servicios han aumentado significativamente el número de

6 Respecto a ello, advierte M. Castells (2002) que se debe abandonar el viejo paradigma de Clark basado en la distinción entre sectores primarios, secundarios y terciarios ya que tal distinción se ha convertido en un obstáculo epistemológico para la comprensión de nuestras sociedades.

7 Para un mayor desarrollo de este debate y una refutación a los posindustrialistas, véase M. Castells (2002). 
ocupaciones con condiciones laborales precarias y territorios sin mayores niveles de desarrollo.

Retomando nuestro análisis, los CCs pueden ser calificados como servicios a la producción o servicios empresariales. La vertiginosa expansión de este tipo de servicios está ligada en Argentina a procesos de desintegración vertical y deslocalización ${ }^{8}$ : las empresas de los países centrales exportan actividades de baja jerarquía intensiva en mano de obra. La posibilidad de almacenar y/o transmitir información simultánea gracias al carácter "líquido" de ésta, que puede ser enviada a través de redes digitales, sostiene esta dispersión geográfica de los CCs, es decir, son empresas que al contar con la capacidad de transmitir su producción en tiempo real, pueden instalarse en espacios descentralizados. La cara de este primer proceso es la multiplicación de sites de empresas transnacionales ${ }^{9}$ que se instalan en Argentina. A escala doméstica se debe observar que existen CCs nacionales que también han crecido de manera sostenida, que crecen al amparo del mayor nivel de actividad del mercado interno, aunque son de menor envergadura en cuanto a facturación y tamaño que las transnacionales ${ }^{10}$.

En definitiva, a nivel territorial, algunas actividades del sector servicios pueden prescindir de la cercanía entre productor y consumidor: en nuestro caso, no es necesaria en los CCs dicha cercanía (dicha proximidad sí es central, por ejemplo, en actividades gastronómicas o de turismo) ya que el objeto de venta es inmaterial, su contenido es información procesada. De esta manera, los servicios que consisten en información se pueden comercializar a distancias cada vez mayores: esta característica es portadora de varios efectos.

En primer lugar, significa que estas actividades se pueden localizar prescindiendo del número local de consumidores, o del poder de compra de la localidad donde se instalan.

En segundo lugar, se puede prescindir de eslabonamientos territoriales sólidos con la cadena de clientes - proveedores. El ejemplo brindado por S. Lash y J. Urry (2004) para la localidad inglesa de Swindon da cuenta de esta realidad: sólo el 14\% de los grandes establecimientos del sector privado en servicios tenía sus abastecedores principales en proveedores locales, mientras que un $39 \%$ recurría a fuentes internacionales.

8 La deslocalización de la producción, es decir, llevar tareas a otros puntos geográficos separados del establecimiento o casa matriz, está amparado en la posibilidad de disociación productiva que permite, por ejemplo, que las empresas desarrollen la relación con el cliente desde otro lugar diferente al de su capital de origen: en este caso se necesita que dicho lugar elegido para realizar sólo esa fase tenga la tecnología adecuada a ese proceso y no al del total del proceso productivo más complejo (López, Ramos, Starobinsky, 2009). Esta característica es central para comprender la posibilidad de varias provincias en Argentina de gestionar exitosamente la instalación de CCs.

9 Los sites son las plataformas desde donde los CCs brindan soporte a las compañías en base a obtener y brindar información de su cartera de clientes. El costo por hora del operador telefónico es la variable clave que determina la instalación de empresas, implica cerca del $70 \%$ de los costos totales de funcionamiento de un site. Dichos costos laborales -medidos en dólares- se reducen drásticamente.

10 Consultora Claves. El mercado de CC en Argentina hoy. Tendencias y oportunidades. VII Congreso

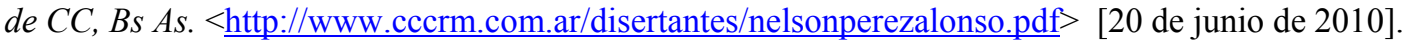

En el informe se destaca que la diferencia entre facturación y tamaño se explica a partir que las multinacionales atienden principalmente la demanda de clientes internacionales y grandes clientes locales, teniendo la posibilidad de exportar, cobrar en dólares y cubrir una demanda de mayor escala. 
En tercer lugar, se observa que el proceso de producción incluye elementos producidos en lugares diferentes y en empresas distintas, ensamblados con propósitos y para mercados específicos. Así, los territorios quedan inmersos en redes o tramas productivas globales a partir de alianzas entre empresas, de unidades descentralizadas de cada una de ellas, de pymes que se conectan con empresas más grandes.

En definitiva, las características territoriales observadas dan cuenta: a) que ciertas actividades del sector servicios tienen la posibilidad de "desengancharse" de las fases del ciclo económico local (es decir, dependen en escasa proporción de la demanda del mercado interno y por tanto no lo afectan sus ciclos recesivos) ya que pueden exportar al mercado global y b) ciertas actividades del sector servicios pueden no tener o tener escasas externalidades positivas sobre el territorio local donde se instalan, por los escasos eslabonamientos detallados más arriba.

Tanto los debates actuales de la sociología urbana como de la sociología de la globalización se materializan al abordar la historia y características de la actividad de $\mathrm{CCs}$, así como sus patrones de localización territoriales. Entonces pasemos a observar con mayor detalle sus especificidades.

\section{SURGIMIENTO Y CARACTERÍSTICAS ESPACIALES DE LA ACTIVIDAD DE LOS CCs}

\section{Surgimiento de los CCs a nivel mundial}

El concepto del telemarketing nace en los años sesenta en los Estados Unidos ${ }^{11}$ e implica una relación telefónica de la empresa con el cliente, en principio para vender un producto o servicio o bien crear y/o afianzar lazos de fidelidad entre ambos.

Esta tendencia se fue expandiendo a Europa, donde Gran Bretaña, Alemania y Francia siempre tuvieron una larga tradición de marketing y venta a distancia. Posteriormente Irlanda y Holanda descubrieron las ventajas de ofrecer incentivos para la instalación de centros de llamados en sus territorios y se transformaron en líderes de la actividad multilingüe ${ }^{12}$. Como se observa, es una actividad que nace en los países denominados centrales o desarrollados y cumpliendo una función de apoyo para la venta de productos. Más adelante, la actividad se ha reconfigurado, y ha mutado de carácter accesorio o de apoyo de ventas para determinados tipos de industria, hasta convertirse en la era actual en una actividad masificada sustentada en el desarrollo de las TICs ${ }^{13}$.

11 Revista Contact Centers, Nro 6.

$<$ http://www.ddm.com.ar/Publicaciones 144 Telemarketing-crisis-u-oportunidad.html $>\quad\left[\begin{array}{lll}15 & \text { de }\end{array}\right.$ noviembre de 2010]. Habitualmente se cita una acción de Ford Motors como la primera gran campaña de telemarketing de la historia. Se contactaron 20 millones de hogares, a razón de 1 millón por semana, para preguntarles sus intenciones de compra de vehículos. Se contrataron y capacitaron 15.000 trabajadores para que realizaran los llamados desde sus hogares. Unos años más tarde la actividad se vio acelerada por la aparición de los números de cobro revertido automáticos (0800), que disparó la industria del telemarketing receptivo por todo EEUU.

12 Idem anterior.

13 Véase Micheli (2004) 
Actualmente, se impone la tendencia de deslocalización en CCs, lo que ha modificado geográficamente la distribución de los sites. La variedad de destinos se ha incrementado significativamente, sumándose a los destinos iniciales mencionados, geografías tan variadas como países sudamericanos, centroamericanos, India, Malasia, Filipinas, Sudáfrica, entre otras.

Respecto al crecimiento y la realidad de Latinoamérica, se destaca como un espacio de enorme recepción de inversiones en esta actividad en la última década. Las figuras que mostramos a continuación dan cuenta de la dinámica de crecimiento (véase Figura 1) y la participación de cada país latinoamericano en este negocio (véase Figura 2). En el caso de los tres principales países en la actividad de CCs en latinoamérica (Brasil, México y Argentina) se destacan algunas características comunes que explican el surgimiento y fuerte instalación de esta actividad: la apertura de sus economías en la década de los '90, transnacionalización de sus empresas y la acción estatal como actor decisivo en la instalación de este agente económico. A estos patrones comunes se suman las particularidades propias de cada país ${ }^{14}$.

14 Para el caso del surgimiento en México es muy ilustrativo el ejemplo que J. Micheli (2004, pp. 21-22) ofrece: "en los años ochenta, Telmex era la empresa pública de telefonía monopólica (...). En 19901991, la empresa Telmex fue privatizada y adquirida mayoritariamente por el Grupo Carso. Así, Telmex creó Teletech con la finalidad de generar sus propias llamadas y retener o recuperar a sus clientes. Posteriormente, Telmex se expandió en un breve lapso hacia el negocio de Internet, asociándose con Prodigy y MSN, y el de computadoras, asociándose con Dell. En este nuevo campo, fue vital el servicio de telemarketing, tanto para promocionar como para atención al cliente. (...) Otro CC de menor tamaño digno de mención como ejemplo de la apertura económica es Telmark. Su evolución marca otra forma característica del desarrollo de estas empresas. Creada como pequeña empresa familiar a inicios de los '90 aprovechó la repentina expansión del mercado causada por la apertura financiera y bancaria, la cual implicó un cambio de propiedad de los bancos tradicionales mexicanos vendidos a bancos internacionales, así como la llegada de nuevos bancos. En ese contexto se desató una guerra de venta de tarjetas de crédito donde el instrumento de telemarketing fue imprescindible".

Para el caso de Brasil véase M. Oliveira Junior (2005) donde se detalla que la expansión de CCs en Brasil se dio gracias a dos factores esenciales: la liberalización de las telecomunicaciones ocurridas en 19971998 y la reglamentación de una ley de Protección al Consumidor en 1990, donde los consumidores accedieron al derecho por ley de recibir información clara en productos y servicios que adquieran, lo que determinó una gran oferta de servicios de CCs. 
Figura 1.

Giro del negocio del sector CCs en América Latina (MUSD)

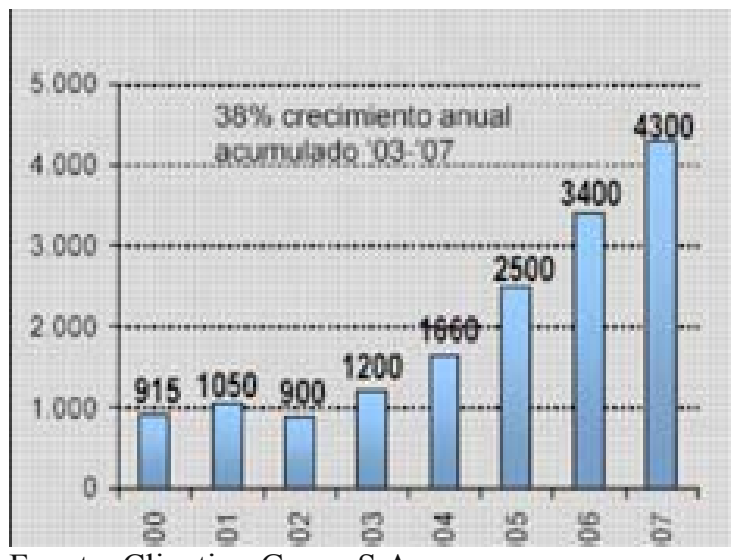

Fuente: Clienting Group S.A.

Figura 2.

Market share por país de América Latina (2007)

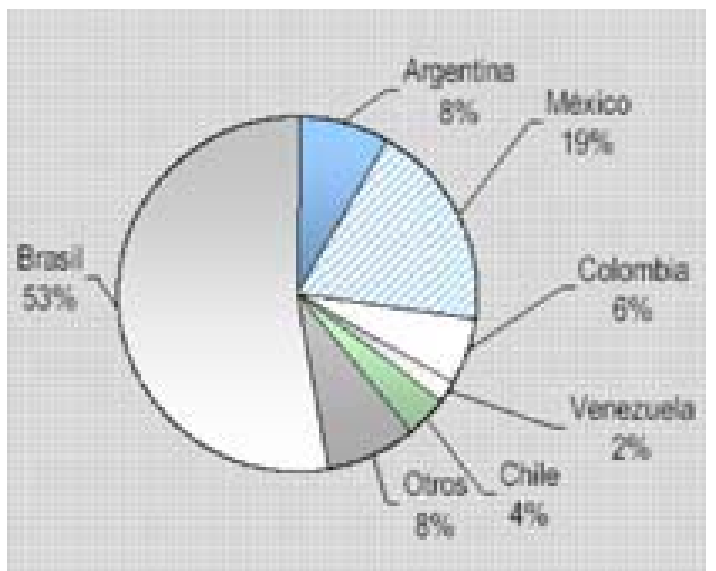

Fuente: Clienting Group S.A.

\section{Los CCs en Argentina}

En Argentina, en los años noventa se comienza a configurar el mercado de oferta de CCs, si bien el comienzo de operaciones relacionadas con la actividad ocurrió a fines de los años ochenta y consistió en estrategias de venta por teléfono: las primeras actividades de telemarketing están asociadas un importante banco norteamericano (Citibank) que a mediados de los ochenta decidió ofrecer tarjetas de créditos a un listado potencial de clientes de alto consumo.

Recién en la segunda mitad de la década del noventa el mercado se fue depurando e, incentivados por la privatización de las telecomunicaciones, que confluye con el auge de esta herramienta en las empresas de los países más desarrollados y las inversiones en nuevas redes de telecomunicaciones, se desarrollan por un lado, agencias profesionales 
que tercerizan principalmente servicios de telemarketing, y por otro lado, los CCs internos o in-house.

En este periodo también se gestó un primer proceso de desnacionalización de agencias siguiendo la misma tendencia que se manifestaba en otros sectores de la economía. Esta primera etapa de crecimiento de los CCs instalados muestra una expansión en el año 2000 , producto de la desregulación de las telecomunicaciones de larga distancia y casi simultáneamente de telefonía celular: se legisla "a medida" de compañías extranjeras privadas de telecomunicaciones, que son las que producen inicialmente una enorme demanda de CCs.

En esta etapa la acción del estado nacional es decisiva para posibilitar el crecimiento de los CCs nacionales y la entrada de empresas extranjeras en fronteras argentinas a partir de la privatización del servicio de telecomunicaciones y posterior desregulación del sistema, con el dictado de normas de regulación laboral flexibles y con marcos regulatorios que homologan reglamentaciones que rigen a escala global (por ejemplo, leyes de protección de datos personales). Luego, el mantenimiento de un tipo de cambio altamente favorable ha beneficiado a CC multinacionales exportadoras de servicios y se termina así de "instalar" un nuevo agente económico.

Luego de la devaluación se masifica el negocio de deslocalización de servicios. Ya existían en el país algunas grandes empresas multinacionales de CCs (Teletech, Teleperformance, Atento) que si bien se habían radicado para dar servicio local a sus clientes globales instalados aquí, aprovecharon los niveles salariales deprimidos y el alto desempleo posdevaluatorio y comenzaron a exportar servicios ${ }^{15}$.

En esta etapa -luego de la devaluación-, con un sector más consolidado, los estados provinciales son los que lideran las estrategias regulatorias y las orientan hacia la atracción de empresas de CCs: ello acelera la instalación de sites en el interior de Argentina. Esta nueva distribución territorial es producto entonces de una estrategia estatal viabilizada a través de acciones, decretos y leyes que incluyen: exenciones impositivas, donación de infraestructura edilicia, subsidios parciales en los salarios de los primeros años de actividad, capacitación de personal costeada por el estado, bonificación de cargas laborales, aportes para equipamiento y oficinas, entre otras ventajas otorgadas para que los CCs se instalen en su territorio.

\section{Características y funcionamiento de la actividad de CCs}

Los CCs son herederos de las nuevas formas de gestión en la relación entre empresasclientes y de la revolución en informática y comunicaciones que permite implementar tecnología en almacenamiento, procesamiento y transmisión de información, integrando computación, telefonía e Internet. Elementos tecnológicos y organizacionales se

15 Este breve recorrido histórico por la historia de los CCs en Argentina, indispensable para explicar la configuración actual del mercado de CCs, está elaborado en base a extractos de una entrevista a Salvador Filiba - Presidente Honorario de la Asociación de Marketing Directo e Interactivo de Argentina (AMDIA). Revista Contact Center Nro. 137. $<$ http://www.ddm.com.ar/Prensa_137_La-industria-de-callcenters-se-pone-los-pantalones-largos.html>. [16 de diciembre de 2010]. 
combinan para lograr una producción adaptada a cada cliente: en este sentido, los operadores telefónicos deben encarar cada proyecto (o campaña en la jerga de los CCs) atendiendo a los cambios o nuevos requerimientos que cada uno implica: un mismo operador al mismo tiempo puede vender tarjetas de crédito, como atender a clientes insatisfechos, o hablar en español neutro e inglés en una misma jornada laboral. La flexibilidad de la mano de obra opera junto a la flexibilidad tecnológica, al utilizar distintos software, marcación predictiva, la distribución automática de llamadas, lo que permite que los mismos medios de producción y de mano de obra brinden diferentes servicios en un mismo CC.

Se debe subrayar que en Argentina los CCs encuentran recursos humanos calificados, con importantes dotaciones -al menos para el contexto regional- de jóvenes con secundario completo y estudios universitarios, bilingües y con acceso tecnológico. Según datos del sector aproximadamente el 70\% de los empleados de los CCs tienen entre 18-24 años, son en su mayoría estudiantes universitarios y mujeres ${ }^{16}$. Este perfil garantiza la competencia comunicacional que requiere el negocio (discursos de presentación, modales de corrección en el trato, etc. $)^{17}$.

Pero vayamos a presentar cómo se compone toda la estructura de la actividad, pues ello es central para comprender sus características territoriales de localización $\mathrm{y}$ funcionamiento.

En primer lugar, debemos entender qué sectores de la economía demandan los servicios de CCs y qué tipo de servicios solicitan. La demanda se encuentra fuertemente concentrada en grandes empresas -nacionales y multinacionales- y los principales sectores demandantes son: telecomunicaciones (compañías de telefonía fija y móvil, medios de comunicación), servicios financieros (bancos, compañías de seguros, tarjetas de crédito), y otros servicios públicos y privados (organismos de gobierno, ONGs, compañías informáticas, empresas de entretenimiento y turismo, entre otros). Entre estas grandes empresas la tendencia es hacia la tercerización del servicio de CCs (aproximadamente el 30\% tienen CCs propios, el resto lo terceriza) ${ }^{18}$.

Estos sectores demandan especialmente servicios de ventas y de atención al cliente. Sin embargo, estas modalidades no agotan la enorme heterogeneidad de demanda de servicios de CC: se suman a los ya detallados, servicios de soporte técnico, de cobranzas, operaciones bancarias, encuestas telefónicas, $\mathrm{BPO}^{19}$, entre otras. Para responder a la demanda, según sea el negocio principal al que se dedique el $\mathrm{CC}$, se necesitarán estrategias de especialización o masividad; tipos de llamados entrantes o salientes, cantidad de posiciones ${ }^{20}$, distinto tipo de sofisticación en soluciones tecnológicas y

16 En Córdoba el $60 \%$ de los operadores telefónicos son mujeres. Diario La Nación on line.. $<$ http://www.lanacion.com.ar/774512-una-voz-argentina-en-el-telefono $>$ [3 de diciembre de 2010]

17 Para un mayor detalle de las competencias necesarias para el puesto de trabajo véase Micheli (2004)

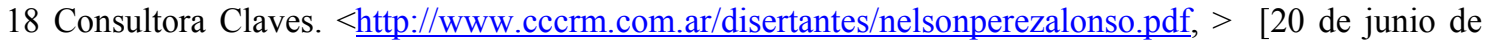
$2010]$.

19 El Business Process Outsourcing (BPO) es un servicio - no masivo aún en Argentina- desde donde los CC -a partir de la administración y análisis de bases de datos de clientes- realizan tareas más sofisticadas de monitoreo y fidelización que no necesariamente incluyen el contacto directo con el cliente.

20 Se llama posición a cada sitio de trabajo con, al menos, computadora, software y hardware específicos. 
calificación de operadores telefónicos. Por tanto, los CCs no deben ser analizados como una realidad homogénea en cuanto a su organización interna.

En base a la coyuntura económica iniciada el año 2002 se observa una marcada tendencia a la extranjerización y alta concentración de los CCs. En un estudio reciente se detalla que menos de diez empresas concentran las operaciones del mercado local y no más de seis firmas lideran la exportación, mientras que las cinco empresas líderes del sector son subsidiarias de grupos multinacionales (del Bono y Henry, 2009). Las empresas extranjeras y multinacionales que lideran este sector tienen la posibilidad de hacer negocios con un mercado local en crecimiento en los últimos años, y al mismo tiempo exportar servicios (lo hacen principalmente a Estados Unidos y Europa -especialmente a España- $)^{21}$. Por la posibilidad de captación de clientes internacionales, mayor tecnología y adaptación a requerimientos globales -por ejemplo, medidas de seguridad informática costosas-, la exportación de servicios es cubierto casi exclusivamente por empresas multinacionales; los CCs de capitales nacionales operan para el mercado doméstico, aunque sufren una competencia cada vez mayor. Los datos cuantitativos sobre facturación muestran que las multinacionales concentran el $60 \%$ del facturado total del sector, las locales un $33 \%$ y las mixtas un $7 \%^{22}$. Coherentemente con esas cifras, las diez empresas de mayor tamaño según cantidad de empleados, son Action Line, Atento, Teleperformance, Teletech, Apex Sykes, Telecom, ACS Multivoice, IBM, ICT Services y Siemens: todas de origen extranjero ${ }^{23}$.

Finalmente, en cuanto a los proveedores necesarios para llevar adelante el negocio: los principales son las compañías telefónicas y la tecnología empleada ${ }^{24}$. Se destaca que son principalmente de origen extranjero los proveedores en las tecnologías de mayor valor agregado (telecomunicaciones, producción de software, dispositivos telefónicos) y de origen local los proveedores de tecnologías menores (por ejemplo, son empresas nacionales quienes fabrican o reparan auriculares o proveen de mobiliario). Además, el avance de CCs posibilitó la creación de proveedores específicos hacia la actividad: carreras universitarias con orientación a la administración de CCs, consultores en selección de operadores telefónicos, empresas de certificación de normas de calidad específicas, entre otros. Para estas últimas tareas pueden encontrarse proveedores locales.

21 En 2009, Europa es el principal lugar de exportación con el 55\% del negocio, seguido de EEUU con el 33\% y América Latina con el 9\% (Fuente: Agrupación Centros de Atención al Cliente de Cámara Argentina de Comercio).

22 Consultora Claves. <http://www.cccrm.com.ar/disertantes/nelsonperezalonso.pdf, > [20 de junio de 2010].

23 Consultora Convergencia Research (2008), CCenters 2007, Bs As, p. 4, en Del Bono y Henry (2009)

24 Como bien dice un gerente de sistemas de un CC pequeño (menos de 50 empleados) ubicado en la ciudad de Bs. As.: "El proveedor clave es el de comunicaciones tanto en gasto económico como en funcionamiento porque si a vos se te caen las líneas telefónicas, tu negocio es llamar por teléfono, vos no podés llamar (...) La tecnología también es clave. A diferencia de lo dicho para las comunicaciones, vos con computadoras viejas podés trabajar, sin embargo vos cargás todo en una máquina que, si anda lenta, hace perder plata a la empresa, se hacen menos llamadas, además que los operadores telefónicos se ponen de mal humor". 


\section{EXPANSIÓN TERRITORIAL DE LA ACTIVIDAD DE CCS EN ARGENTINA}

La primera etapa de expansión territorial de la actividad está caracterizada por una marcada concentración geográfica tanto de CCs como de clientes y proveedores. Es una actividad aún no predominantemente transnacionalizada y que está orientada a cubrir la demanda del mercado local. Dicha concentración tiene sede en la Ciudad Autónoma de Buenos Aires (CABA), que es la pionera en esta actividad, pues varios CCs se habían instalado allí antes del 2002, aprovechando una abundante mano de obra, infraestructura tecnológica desarrollada y proveedores tecnológicos ya instalados.

La segunda etapa se define por una mayor dispersión geográfica, producto de estrategias empresariales de maximización de rentabilidad por abaratamiento de costos laborales, mayores subsidios y exenciones impositivas, además de búsqueda de menor capacidad organizativa de los trabajadores ${ }^{25}$. Con estas claves explicativas se interpreta la llegada masiva de CCs al interior del país: un análisis histórico de la evolución de empleo directo generado muestra una sistemática pérdida relativa de puestos de trabajo en CABA frente al crecimiento del resto de las plazas, como se observa en el cuadro 1 .

\section{Cuadro 1. Empleos directos generados por CCs tercerizadores y participación porcentual por provincia, en Argentina en el período 2002-2010}

\begin{tabular}{|c|c|c|c|c|c|c|c|c|c|c|c|c|}
\hline \multirow[b]{2}{*}{ Año } & \multirow{2}{*}{$\begin{array}{l}\text { Empleos generados y } \\
\text { participación } \\
\text { porcentual en el país }\end{array}$} & \multicolumn{11}{|c|}{ Principales espacios de localización de CC } \\
\hline & & CABA & $\begin{array}{l}\text { Cór } \\
\text { doba }\end{array}$ & $\begin{array}{l}\text { Prov. } \\
\text { Bs As }\end{array}$ & $\begin{array}{l}\text { Tucu } \\
\text { mán }\end{array}$ & $\begin{array}{c}\text { Santa } \\
\mathrm{Fe}\end{array}$ & Salta & $\begin{array}{l}\text { Men } \\
\text { doza }\end{array}$ & Chaco & $\begin{array}{l}\text { San } \\
\text { Luis } \\
\end{array}$ & $\begin{array}{c}\mathrm{La} \\
\text { Pampa }\end{array}$ & $\begin{array}{r}\text { TO } \\
\text { TAL }\end{array}$ \\
\hline \multirow{2}{*}{2002} & Empleos (absolutos) & $\mathrm{S} / \mathrm{d}$ & $\mathrm{S} / \mathrm{d}$ & $\mathrm{S} / \mathrm{d}$ & - & - & - & - & - & - & - & 5.000 \\
\hline & Participación en país (\%) & $\mathrm{S} / \mathrm{d}$ & $\mathrm{S} / \mathrm{d}$ & $\mathrm{S} / \mathrm{d}$ & - & - & - & - & - & - & - & \\
\hline \multirow{2}{*}{2003} & Empleos (absolutos) & 9.500 & 2.000 & 500 & - & - & - & - & - & - & - & 12.000 \\
\hline & Participación en país (\%) & $80 \%$ & $16 \%$ & $4 \%$ & - & - & - & - & - & - & - & \\
\hline \multirow{2}{*}{2004} & Empleos (absolutos) & 12.100 & 5.600 & 1.100 & - & 1.130 & - & 70 & - & - & - & 20.000 \\
\hline & Participación en país (\%) & $60 \%$ & $28 \%$ & $6 \%$ & - & $5,6 \%$ & - & $0,4 \%$ & - & - & - & \\
\hline \multirow{2}{*}{2005} & Empleos (absolutos) & 16.200 & 9.000 & 3.300 & - & 1.350 & - & 150 & - & - & - & 30.000 \\
\hline & Participación en país (\%) & $54 \%$ & $30 \%$ & $11 \%$ & - & $4,5 \%$ & - & $0,5 \%$ & - & - & - & \\
\hline \multirow{2}{*}{2006} & Empleos (absolutos) & 24.750 & 14.400 & 4.000 & - & 1.050 & 225 & 575 & - & - & - & 45.000 \\
\hline & Participación en país (\%) & $55 \%$ & $32,5 \%$ & $8,5 \%$ & - & $2 \%$ & $0,5 \%$ & $1,5 \%$ & - & - & - & \\
\hline \multirow{2}{*}{2007} & Empleos (absolutos) & 23.100 & 22.550 & 4.950 & - & 1.650 & 550 & 2.200 & - & - & - & 55.000 \\
\hline & Participación en país (\%) & $42 \%$ & $41 \%$ & $9 \%$ & - & $3 \%$ & $1 \%$ & $4 \%$ & - & - & - & \\
\hline \multirow{2}{*}{2008} & Empleos (absolutos) & 26.614 & 29.183 & 6.916 & 2.973 & 2.170 & 1.015 & 1.309 & 60 & - & - & 70.240 \\
\hline & Participación en país (\%) & $37,9 \%$ & $41,5 \%$ & $9,8 \%$ & $4,2 \%$ & $3,1 \%$ & $1,5 \%$ & $1,9 \%$ & $0,1 \%$ & - & - & \\
\hline \multirow{2}{*}{2009} & Empleos (absolutos) & 23.348 & 28.063 & 6.790 & 3.577 & 2.088 & 1.330 & 1.303 & 60 & 90 & - & 66.649 \\
\hline & Participación en país (\%) & $35 \%$ & $42,1 \%$ & $10,2 \%$ & $5,4 \%$ & $3,1 \%$ & $2 \%$ & $2 \%$ & $0,1 \%$ & $0,1 \%$ & - & \\
\hline \multirow{2}{*}{2010} & Empleos (absolutos) & $\mathrm{S} / \mathrm{d}$ & $\mathrm{S} / \mathrm{d}$ & $\mathrm{S} / \mathrm{d}$ & 6.000 & $\mathrm{~S} / \mathrm{d}$ & $\mathrm{S} / \mathrm{d}$ & $\mathrm{S} / \mathrm{d}$ & 1.380 & 190 & 140 & $\mathrm{~S} / \mathrm{d}$ \\
\hline & Participación en país (\%) & $\mathrm{S} / \mathrm{d}$ & $\mathrm{S} / \mathrm{d}$ & $\mathrm{S} / \mathrm{d}$ & $\mathrm{S} / \mathrm{d}$ & $\mathrm{S} / \mathrm{d}$ & $\mathrm{S} / \mathrm{d}$ & $\mathrm{S} / \mathrm{d}$ & $\mathrm{S} / \mathrm{d}$ & $\mathrm{S} / \mathrm{d}$ & $\mathrm{S} / \mathrm{d}$ & $\mathrm{S} / \mathrm{d}$ \\
\hline
\end{tabular}

Fuente: Agrupación Centros de Atención al Cliente de Cámara Argentina de Comercio. Elaboración propia $\mathrm{S} / \mathrm{d}$ Sin datos

El avance de CCs en el interior del país se sostiene en multinacionales que se instalan allí con estrategias empresariales de carácter global, lo que les permite jerarquizar territorialmente el proceso productivo, deslocalizando tareas de menor valor agregado,

25 Para observar las prácticas del sindicato hegemónico en la actividad (comercio) y sus características poco representativas de los trabajadores de CCs, véase S. Garro (2008) y A. Del Bono y L. Henry (2008). 
poco especializadas y de baja capacitación. Pero si bien llegan CCs al interior del país, permanecen clientes y proveedores calificados concentrados geográficamente en CABA y Córdoba -y en menor medida en Rosario-.

Veamos las características puntuales de la distribución territorial de la actividad.

En primer lugar, CABA y Córdoba son los territorios donde esta actividad tiene la mayor cantidad de sites instalados. Luego, donde más empresas existen es la provincia de Bs As, Mendoza, Tucumán, Rosario, Chaco, San Luis y Salta, entre las ciudades con más de una empresa. Tanto en la Patagonia (a excepción de Neuquén) como en zonas del NEA (a excepción de Chaco) y Mesopotamia (a excepción de Entre Ríos) no hay CCs instalados. El fenómeno de exclusión de CCs en esos espacios geográficos se asocia a que no hay aún en esos territorios legislación a medida para la actividad, y se suma a esto un problema de escasa conectividad (especialmente en zona Patagonia).

En segundo lugar, el avance de los CCs en el interior del país se observa primero lentamente y sólo en algunas plazas (Córdoba, Rosario, provincia de Bs. As.); luego se acelera y se suman muchas más provincias (Mendoza, Salta, Tucumán, recientemente Chaco, San Luis, La Pampa) determinando -luego del año 2002- un crecimiento porcentual de nuevas empresas radicadas mayoritariamente en el interior del país.

En tercer lugar cabe destacar que los CCs se localizan en las ciudades principales de cada provincia. En escasas ocasiones se ubican en segundas ciudades (Mar del Plata, Bahía Blanca, San Rafael, Justo Daract, Gral. Pico). En definitiva, la expansión de los CCs es un fenómeno eminentemente urbano, de grandes ciudades, es decir donde hay mayor concentración de población (recursos humanos disponibles) y mayor densidad de infraestructura tecnológica ${ }^{26}$.

Por último, resulta interesante observar diferencias entre CABA y el interior del país en cuanto al tamaño de los CCs y sus capitales de origen. En comparación a CABA, los CCs que se instalan en el interior son de mayor tamaño, y son significativamente predominantes las empresas multinacionales. A nivel tamaño, el caso paradigmático es Córdoba, donde existen menos CCs, pero generan más empleo (no solo relativo, sino absoluto) que en CABA. En cuanto al capital de origen, se pueden nombrar como ejemplo Tucumán y Chaco, con varios CCs allí pero sólo uno de capital nacional.

Si se hace un balance de la distribución territorial, se observa una mayor dispersión geográfica de sites de CCs en el país frente a la concentración muy densa de proveedores (especialmente de alta tecnología y calificación) sólo en CABA, Córdoba y Rosario. Por ello podríamos hablar de una actividad fragmentada en su conjunto. Así, en aquellos tres territorios dinamiza sectores de producción de mayor valor agregado, pero no lo hacen en el resto de las ciudades donde se localizan, donde su externalidad exclusiva es la de

26 Respecto a la infraestructura tecnológica son ilustrativas las palabras de un gerente de sistemas de CC ubicado en CABA: "Vos imaginate que si tenés que tirar cableado de fibra óptica a una casa que está en medio del campo, le tenés que cobrar 10 mil dólares por mes para que te sea redituable, porque hacés una gran inversión en infraestructura para una sola persona. Entonces ¿qué pasa? Yo me puedo poner un call center en Chubut, pero me tengo que manejar con un Speedy de $512 \mathrm{~K}$ de velocidad de banda ancha, eso no sirve para nada, menos para hacer llamadas digitales". 
generar empleo. Igualmente vale aclarar que la dinamización y -en algunos casosespecialización de proveedores en la implementación o desarrollo de productos y/o servicios demandados por los CCs, en ningún caso "lleva" nuevas empresas consigo -al estilo, por ejemplo, de lo que podría hacer el sector automotriz- ${ }^{27}$.

\section{Territorios locales y estrategias de desarrollo: un debate político en torno a la llegada de CCs}

Una de las causas explicativas - la decisiva- de la pérdida relativa de puestos de trabajo en CABA frente al interior del país, es que en CABA las políticas de beneficiar con exenciones impositivas y subsidios han sido más erráticas que en las provincias en los últimos años. En este sentido, es en el interior del país es donde las empresas en mayor medida obtienen beneficios fiscales y de otros tipos.

En los últimos cinco años se ha generado un verdadero efecto "cascada" entre provincias, en cuanto a la legislación ofrecida a la actividad, dando sustento empírico a uno de los efectos de la expansión de los CCs: la emergencia de nuevas formas de competitividad interterritorial (Ciccollela, 1996) por captar tales inversiones ${ }^{28}$. Este proceso nos remite a abordar los debates políticos, pues es donde se plasman discursos acerca de las estrategias de desarrollo en cada territorio local.

En primer lugar, cabe decir que las políticas de exenciones tributarias, diferenciales a nivel territorial, son ampliamente fomentadas por los agentes económicos en su intento de lograr mayor rentabilidad allí donde se instalan. El brazo político de los agentes económicos son las cámaras empresariales de CCs que, a través de sus distintos órganos de difusión (congresos, revistas especializadas), despliegan su capacidad de lobby político. La habitual presión discursiva hacia las instancias estatales pondera la mayor competitividad de otros países o provincias, lo que opera como demanda de menores costos laborales y/o mayores beneficios fiscales que los compensen. Las demandas interpelan tanto al estado nacional como a los estados provinciales. Para ilustrar la presión al estado nacional son habituales frases del siguiente tipo:

"La Argentina es cada vez menos atractiva para los inversores del exterior y, de no mediar medidas oficiales para fomentar la actividad, el país puede quedar fuera del mapa entre los principales players del mundo", "Cuando tenés gobiernos que alientan la industria de Perú y Colombia que compiten con nosotros en offshore fundamentalmente a España, a la industria le quedan pocas herramientas porque no maneja el tipo de cambio, ni las regulaciones, ni los subsidios" y "Durante el primer trimestre del año, la

27 Ilustra bien esta situación la declaración del gerente de Administración y Finanzas de un CC mediano (alrededor de 800 empleados) ubicado en la ciudad de Bs. As.: "...el hecho de que nosotros estemos en Bs As junto al resto de los proveedores y desarrolladores de software y de telefonía y redes, etc, etc, es más producto del macrocefalismo porteño...de todo concentrado acá en Bs As que una externalidad imputable a determinada a esta actividad"

$28 \mathrm{El}$ autor citado se refiere al nuevo paradigma tecnológico productivo como el que "Ha desencadenado una dura puja entre regiones, entre ciudades, todas contra todas a nivel mundial, por la radicación de inversiones, dando lugar a lo que podría denominarse la competitividad interterritorial, en una economía sumamente globalizada" (Ciccollela, 1995, p.48). 
Argentina perdió de ganar 2.000 nuevos puestos de trabajo. La mano de obra quedó en manos de México, Perú, Costa Rica, El Salvador y Filipinas". 29

Mientras que para presionar a los estados provinciales se apela a casos provinciales de "éxito" desde la perspectiva de los agentes económicos como son el caso Córdoba y más recientemente- el caso Tucumán, donde los CCs han obtenido enormes beneficios vía leyes, decretos y subsidios. Como muestra lo siguiente:

“...al ser un sector nuevo a veces cuesta el entendimiento de la dinámica del sector (...) hasta el momento hemos logrado, por ejemplo, que en la provincia de Tucumán entiendan claramente de lo que es capaz el sector; de que en Córdoba sigan, de alguna manera, algunas exenciones impositivas. ",30

Frente al discurso de los agentes económicos, se configuran discursos políticos y acciones estatales. El análisis de ambos tipos de discursos en conjunto, permite visibilizar mecanismos de cooperación y/o conflicto mediante los cuales los actores implicados posibilitan y modelan la instalación de CCs en Argentina. Sin embargo, los discursos varían según las realidades de cada territorio: las capacidades de negociación y de concesiones posibles están enmarcadas por la realidad de cada uno de los espacios locales.

Veamos algunos de los debates políticos que abordan ciertos elementos de desarrollo para los territorios locales, en este caso, en CABA y Córdoba; vale aclararlo, pues estos espacios podrían calificarse como "privilegiados" en tanto poseen un mayor desarrollo socioeconómico en comparación a la mayoría de las provincias argentinas. Una de nuestras hipótesis es que los territorios "privilegiados" tienen mayores márgenes de acción para discutir estrategias de desarrollo de mayor alcance y no reducir sus debates a los beneficios impositivos necesarios para atraer la llegada de $\mathrm{CCs}^{31}$ (más adelante veremos en detalle las características de este debate en territorios "no privilegiados" lo que permitirá un análisis más amplio de las capacidades de negociación).

Durante el debate parlamentario del año 2005 en la legislatura cordobesa por el proyecto de ley de beneficios impositivos para que los CCs se instalen en su territorio, una de las críticas que surgieron hacia el proyecto es que planteaba la necesidad de exenciones tributarias necesarias para desarrollar de un polo tecnológico en la provincia La idea de este polo había surgido especialmente a partir del inicio del año 2001, cuando distintos actores comenzaron a organizarse para consolidar a Córdoba como

29 Frases extraídas del Informe a cargo de José Romero Victorica (2009) - ejecutivo referente del sector-, Presidente del CC Allus Global y presidente y Fundador de Cámara Empresaria de Servicios y Contactos para Terceros en Córdoba (CESCT).

$<$ http://www.siicex.gob.pe/siicex/resources/sectoresproductivos/Presentaci\%C3\%B3n_PromPeru_ALLUS_2009.pdf $>$. [2 de febrero de 2011].

30 Frases que se extrajeron de una entrevista a Fernando Padrón (2010) -otro referente del sector-, Presidente del CC ActioLine. Revista Contact Center Nro 143. $<$ http://www.contactcentersonline.com/revista.php?IdRevista=143 $>$. [21 de marzo de 2011].

31 Esta característica no implica que efectivamente las estrategias de desarrollo se lleven a la práctica o sean las más beneficiosas para el territorio local: eso depende de coyunturas políticas (gobiernos de turno y mayorías parlamentarias) junto a coyunturas económicas (crisis, crecimiento, desocupación, pleno empleo). 
destino de inversiones en el sector de las TICs. Dentro de un programa de fomento a la creación de este polo tecnológico -que incluyó regímenes de promoción industrial para la radicación de industrias tecnológicas y desarrollo de software- se terminó incluyendo a los CCs como parte de este polo cuando inicialmente fue pensado para recibir empresas de software y electrónica, lo que fue posible dada la presión ejercida por las cámaras empresariales de CCs y la rápida asistencia de los gobiernos provinciales y municipales para incorporarlos. Una de las voces críticas a esta concepción de "polo tecnológico" fue la del legislador Fernández del Bloque Frente Nuevo, que en su intervención parlamentaria declaraba:

“... el señor Secretario de Industria comentó el antecedente de Irlanda, pero no se corresponde con la verdad. En Irlanda se desarrolló el software y el hardware a partir del apoyo de la Comunidad Económica Europea con cuantiosos fondos que fueron destinados a Irlanda como zona desfavorable y capitales provenientes de la costa este de Estados Unidos. Radicaron capital genuino para inversión en estos sectores, acompañado con los servicios; no se crearon los servicios para que después surgieran las otras empresas $" 32$

Recientemente en CABA se ha intentado legislar para ofrecer más facilidades a los CCs para que se instalen en el denominado Distrito Tecnológico (DT), zona que se crea como una iniciativa del gobierno local para fomentar la economía de la ciudad pero también para incentivar la zona sur en cuanto a infraestructura, seguridad. El DT lo que ofrece es una zona libre de impuestos locales, a nivel ciudad, para las empresas privadas del sector de TICs. Sin embargo, aunque se había tomado la decisión desde el gobierno local de incluir a los CCs en los beneficios, luego se dio marcha atrás en la legislatura. Una funcionaria del gobierno, del área del Centro de Atención al Inversor (Ministerio de Desarrollo Económico) resume la situación:

“...cuando se crea la ley, cuando se desarrolló el proyecto, los call centers estaban incluidos bajo la ley. Cuando esa ley fue aprobada, se llevo a cabo todo el proceso en la legislatura, eso anduvo bien. Luego, el inciso $K$ del artículo 2 de la ley se derogó... ¿por qué? Porque los call center no generan un valor agregado en cuanto a la actividad, por eso se derogó (...) se hizo todo un estudio, no fue al azar...en realidad los call centers el tema que tienen es que si bien funcionan acá en Buenos Aires, son empresas generalmente de Estados Unidos, las que van a recibir este cambio. No generarían una promoción para la zona sur, que es el objetivo principal, el "premio" se lo lleva la empresa que está en Estados Unidos, en Londres, y no en Argentina como nación, o en Buenos Aires como ciudad"

Hemos citado ambas intervenciones, pues nos interesan en tanto visibilizan los límites de la actividad de CCs y nos alertan sobre los alcances de la captación de este tipo de inversiones como única estrategia de desarrollo posible para los territorios locales urbanos.

32 Diarios de Sesiones de la $14^{\mathrm{a}}$ Reunión $-12^{\circ}$ Sesión Ordinaria del Poder Legislativo de la Provincia de Córdoba del 13 de Abril de 2005, pp. 710-721. Diarios de Sesiones de la Legislatura de la Provincia de Córdoba. <http://www.legiscba.gov.ar/info_sesiones.asp/>. [2 de marzo de 2011]. 
A continuación se describe el proceso de localización de CCs en la provincia argentina de Chaco. Este caso busca ilustrar concretamente lo expuesto hasta aquí, en un espacio local con bajos índices de desarrollo socioeconómico en Argentina que, sin embargo, logra captar actividades globales.

\section{La localización de CCs en la ciudad de Resistencia}

Chaco es una de las provincias más pobres de Argentina, situada geográficamente en el nordeste del país: en un estudio del año 2006 se detalla que Chaco posee unas de las tasas de pobreza e indigencia más altas de Argentina (60 y 35\% respectivamente) que, sin embargo, convive junto a una de las tasas de desocupación más reducidas, el 5\%. Tres características del empleo de la población pueden explicar esta aparente paradoja: las bajas tasas de salarios, la mayor cantidad de personas que tiene que mantener cada trabajador y la alta proporción de desocupación “escondida” en la provincia. ${ }^{33}$.

Resistencia es la capital del Chaco, su ciudad más poblada y su principal centro económico; las fuentes centrales de ingresos para la población provienen de la administración pública y de un importante sector logístico, ya que su ubicación y accesos (ferroviario, aeroportuario, carretero y fluvial) la convierten en un emplazamiento ideal para centros de distribución y transferencia de toda la región nordeste del país. Geográficamente, su proximidad con la ciudad de Corrientes permite la integración de ambas en forma de conurbano, lo que las convierte en un importante centro metropolitano del Nordeste argentino.

Resistencia no escapa a la realidad provincial, a sus problemas sociales y de empleo. En este contexto, la inserción laboral de sus jóvenes es compleja, justamente el segmento que en mayor medida utilizan como operadores telefónicos los CCs. En un reciente estudio se detalla que:

“...el mayor grado de vulnerabilidad de los jóvenes (en el Gran Resistencia) quedaba también en evidencia en las estimaciones sobre la variación de las tasas de ocupación ya que eran quienes presentan una caída mucho más pronunciada que en los restantes grupos etarios. Asimismo, algunos indicadores de la calidad de las ocupaciones obtenidas (tipos de contratación, calificación ocupacional, tiempo de trabajo, acceso a beneficios sociales y el nivel de ingresos) daban cuenta de la desfavorable situación en la que se encontraban" (Barbetti, Pablo, 2003, p. 2)

En el caso de Chaco, el inicio de servicios de sus CCs es el más reciente comparado con el resto de las provincias del país, con un crecimiento exponencial durante el año 2010 en una actividad inédita en la provincia hasta el año 2008. Todas las empresas se radican en la ciudad de Resistencia. Cuatro son los CCs que operan en la ciudad: Telcel, Voicenter, y las dos que lideran el mercado, Atento (del Grupo Telefónica de capitales españoles) y Allus (capitales norteamericanos). Las últimas dos empresas se radican a comienzos del

33 Estudio realizado por el Consejo Económico y Social de la Provincia de Chaco (CONES) en base a la Encuesta Permanente de Hogares (EPH). <http://www.coneschaco.org.ar/newsitio/libro/AnexoA1ProblemasdeEmpleodelChaco.pdf/>. [20 de abril de 2011]. 
año 2010 y se dedican especialmente al rubro ventas y servicios telefónicos de atención al cliente -los servicios que necesitan menor calificación en sus operadores telefónicos-. Entre ambas empresas generan aproximadamente 1.300 puestos de trabajo, alrededor del $90 \%$ de los empleos que generan los CCs en toda la provincia.

Respecto a la llegada de Atento, se destaca en los medios provinciales que el CC crea 700 puestos de trabajo y en una segunda etapa preveen incorporar 500 empleados más, sin dar mayores detalles al respecto. También con la llegada de Voicenter unos meses atrás se anuncia la creación de 120 puestos de trabajos y se prevee -en el corto plazo- duplicar la mano de obra.

Los anuncios y planes de las empresas van en perfecta sintonía con las promesas estatales. En el año 2008, el gobernador Capitanich concurre a la inauguración de Telcel y promete:

“...crear unos tres mil puestos de trabajo en Chaco relacionados al negocio del contacto en los próximos tres años y la próxima apertura de un Centro de Formación Profesional en Contact Center del Nordeste",34

En el año 2010, con el arribo de la firma Allus, la prensa local publica declaraciones del ministro de economía chaqueño Eduardo Aguilar quien asegura respecto a los CCs

“...es una industria floreciente porque estas empresas tienen la capacidad de creación masiva de puestos de trabajo con inversiones relativamente bajas. El capital por puesto de trabajo es mucho más bajo que en las industrias tradicionales que son de exportaciones de servicios, además tiene capacidad de absorber gran cantidad de mano de obra",35

En el portal web de la provincia se publica:

"Esta gestión ha puesto en marcha el programa más importante de la historia del Chaco en cuanto a incentivos y estímulos para la inversión privada y la generación de empleo y los resultados están a la vista",36

En todos los casos la llegada de estas inversiones es en el marco de amplios incentivos a las empresas que se otorgan mediante leyes para atraer inversiones.

En los debates en la legislatura provincial en el año 2008, la diputada Cristófani (Bloque Alianza Frente de Todos) impulsora junto al poder ejecutivo del proyecto para la radicación de CCs en la provincia señala ${ }^{37}$ :

\footnotetext{
34 Revista Contact Center. $<$ http://www.contactcentersonline.com/novedad.php?IdNovedad=288 $>$. [2 de febrero de 2011].

35 Diario Norte. http://www.diarionorte.com/noticia.php?numero=50236. [29 de octubre de 2010].

36 Portal del gobierno provincial. $<$ http://portall .chaco.gov.ar/pagina?id=8275>. [1 de octubre de 2010]. 37 Se transcriben a continuación la Versión taquigráfica de la reunión Nro.33 Sesión Ordinaria Nro.23 de las Sesiones de la Cámara de Diputados provinciales en Resistencia (Chaco) el 10 de septiembre de 2008. $<$ http://www.legislaturachaco.gov.ar/ActividadesLegislativas/DiarioDeSesiones.aspx >. [29 de marzo de 2011].
} 
"Este tipo de empresa se está instalando en todo el mundo -es "lo que se viene"- y tenemos la posibilidad que (en) la provincia -que carece de grandes industrias como para generar empleos- se permita -de acuerdo con las investigaciones que estuvimos haciendo en otras provincias como Mendoza, Córdoba y la Capital Federal- este primer empleo que facilita, sobre todo a los jóvenes estudiantes, tener un trabajo de poco tiempo que les permita continuar con sus estudios"

Más adelante destaca:

"Podemos desde aquí, el Chaco, prestar servicios a empresas de México, España, de habla hispana, y lógicamente, si tenemos gente que maneje otros idiomas, también podemos prestar un servicio a todo el mundo ,38

Una de las escasísimas voces disidentes al proyecto, el de la diputada Terada (bloque ARI) enuncia:

“...el tema de los CC está estrechamente relacionado con las dificultades que tiene este tipo de instalación como trabajo estresante, en cierto modo dificultoso y en algunos casos no bien reconocido (...) En función de eso, de los antecedentes, del conocimiento y del tratamiento que se hizo en el Congreso de la Nación respecto de este tema puntual donde el bloque del ARI planteo las dificultades para el denominado "trabajo esclavo" que se da en estos centros de llamadas, pero fundamentalmente porque en el artículo 3ro se otorga una exención del ciento por ciento de los Impuestos por Ingresos Brutos y Sellos que me parece en cierto modo excesivo en relación con el resto de los comercios que están obligados a pagar los impuestos, solicito al Cuerpo la autorización para abstenerme de votar este proyecto, 39

En dicha sesión se votan y aprueban, sin modificaciones, todos los artículos de la ley propuesta, con una única abstención, la de la diputada Terada.

La ley ${ }^{40}$ establece un régimen de promoción para las empresas de CC “...destinadas a prestar servicios esenciales a industrias que pueden o no estar radicadas en la Provincia", como taxativamente señala en su artículo 2 do. Por dicha ley quedan eximidas del pago de los impuestos a los ingresos brutos y sellos en un $100 \%$ durante los primeros cuatro años y luego de manera decreciente. Luego, mediante el decreto 3987/08 ${ }^{41}$ y más adelante en el artículo 1ro del decreto $1.058 / 10^{42}$ se comienza a reglamentar la ley y se incluye que los CCs recibirán una compensación por la contratación de mano de obra, la misma equivale a un porcentaje de la remuneración total del personal, no directivo y no

38 Idem anterior, p. 190 (los números de página corresponden a la versión impresa).

39 Idem anterior, p. 191 (los números de página corresponden a la versión impresa)

40 Ley Nro. 6.209, sancionada por la Cámara de Diputados provincial. $<$ http://economia.chaco.gov.ar/index.php?option=com_wrapper\&view=wrapper\&Itemid=146>. $\quad[20 \mathrm{de}$ febrero de 2011].

41 Decreto nro. 3.987 publicado el 26 de noviembre de 2008 en el Boletín oficial de la provincia. $<$ http://portal1.chaco.gov.ar/uploads/boletines/bf14ccfecf7420452456250896be6dc0af3d43b2.pdf $>$. [12 de febrero de 2011].

42 Gobierno de Chaco. $<$ http://economia.chaco.gov.ar/index.php?option=com_wrapper\&view=wrapper\&Itemid $=144>. \quad\left[\begin{array}{ll}6 & \mathrm{de}\end{array}\right.$ febrero de 2011]. 
gerencial, de entre 18 y hasta 30 años de edad, que dichas empresas contraten en la provincia, durante los cuatro primeros años, en una escala que oscilará entre el $5 \%$ y el $30 \%$ del sueldo total, decreciendo según aumente la edad, luego del primer año trabajado, y según el volumen de empleos creados.

Otra ventaja otorgada fue la cesión de parte de las instalaciones de una dependencia del edificio de Desarrollo Social provincial para el funcionamiento operativo de un CC. El artículo 5to del decreto 3.987 lo avala explícitamente: "Las empresas call center, contac center o de alojamiento web podrán recibir facilidades para la locación o cesión en comodato de bienes inmuebles de dominio del Estado". En el artículo 8vo se define que el compromiso de los CC para acceder a los beneficios del artículo 5to sólo será permanecer al menos ocho años en la provincia.

También desde la Agencia de Empleo provincial (dependiente del Ministerio de Economía, Industria y Empleo) se pone a disposición de las empresas bases de datos con la elaboración de perfiles laborales de recursos humanos para que luego la empresa entreviste a las personas registradas, con lo cual se reducen significativamente los costos de selección de personal ${ }^{43}$. Esta tarea, que a priori es asociada al ámbito privado (empresas de recursos humanos y selección de personal) se suple con la iniciativa estatal. La iniciativa privada es débil, lo que se evidencia en que no hemos relevado proveedores de alta tecnología o de alta formación radicados en Chaco.

En este contexto de beneficios fiscales y subsidios, las cámaras empresariales han comenzado a ponderar los esfuerzos del gobierno provincial: elogian en revistas especializadas las nuevas potencialidades en Chaco. Los agentes económicos no parecen tener oposición: no han surgido aún actores políticos locales de peso que se opongan a los amplios estímulos fiscales otorgados ni sindicatos que representen a los operadores telefónicos, que están encuadrados bajo el desventajoso convenio de comercio.

\section{MODELOS DE DESARROLLO EN ESPACIOS URBANOS}

Presentamos a continuación un modelo de desarrollo en espacios urbanos en torno a la llegada de CCs al territorio, elaborado en base a la evidencia empírica.

Hemos construido el concepto de modelo de desarrollo de corto plazo, para dar cuenta de las características centrales que se visualizan a partir del caso chaqueño. Dicho modelo sustenta el avance de CCs exclusivamente por la gestión estatal provincial y sus lineamientos generales pueden resumirse en la estrategia de retención de jóvenes en su ciudad principal (Resistencia).

El rol estatal, en su afán de radicar CCs, realiza incluso tareas habitualmente reservadas a la iniciativa privada (selección de personal, la instalación de sites en edificios públicos, etc.).

43 El mismo Ministro de Economía provincial Aguilar pondera que los requisitos para presentarse a la convocatoria son básicos: tener más de 18 años, secundario completo y disposición para venta y atención telefónica. Diario Norte. <http:/www.sietepuntasdigital.com/2.0/Nota.php?idn=34014>. [21 de noviembre de 2011]. 
Si bien Chaco podría ser considerada una provincia "no privilegiada" en tanto no posee una estructura productiva de magnitud, y desde esta perspectiva puede defenderse la llegada de CCs, bien podría asociar a su estrategia de retención una búsqueda de mayor desarrollo de sus jóvenes, en cuanto a aprendizajes técnicos y capacitación profesional. Se trata de una estrategia estatal que busca inclusión en el mercado laboral fomentando la generación de empleo en los territorios; pero no intenta calificar a sus jóvenes. Es decir, que el estado provincial eroga recursos y energías para que los jóvenes urbanos no emigren a otras ciudades, pero se les ofrece tareas y posibilidades de desarrollo inferiores a sus potencialidades o con baja estabilidad en el tiempo. Si bien es importante el impacto cuantitativo en los empleos generados, se debe instalar con urgencia un debate por la calidad del empleo.

También está ausente en este modelo de desarrollo la incentivación estatal para la llegada de proveedores de mayor calificación para los CCs, que produzcan localmente o que tomen mano de obra local.

Por las características expuestas, lo hemos denominado como modelo de corto plazo, donde los CCs que se instalan son esencialmente multinacionales que no generan externalidades positivas más allá del empleo de baja calificación ofrecido. Al radicarse exclusivamente por los bajos costos en salarios ofrecidos por la economía local, y con la capacidad de relocalizarse en otras plazas, abandonarán la provincia si consiguen salarios aún más deprimidos en otros territorios o cuando el desgaste de la mano de obra le marque un límite a la oferta.

\section{COMENTARIOS FINALES}

En Argentina, así como en los espacios latinoamericanos, es masiva la llegada de inversiones en la actividad de CCs. El proceso de globalización y de apertura de los mercados produjo importantes y heterogéneas transformaciones en los territorios. En este marco, actividades económicas globalizadas y con características digitales como son los CCs se instalan en territorio locales y utilizan mano de obra intensivamente, infraestructura tecnológica, leyes y ventajas fiscales, entre otros atributos ligados a lo local.

Estas características determinan su instalación en espacios urbanos. Si bien dichos espacios -aún los de peores condiciones de desarrollo socioeconómico en los paísesdemuestran capacidad para captar estas inversiones, no logran más que una integración subordinada, en la que dependen de actores globales externos sobre los que no tienen mayor control. En este sentido, las sociedades se configuran alrededor de nuevas desigualdades territoriales. Varios autores han dado cuenta de dichas desigualdades, Castells afirma que:

"La arquitectura de la economía global ofrece un mundo asimétricamente interdependiente" (Castells, 2002, p. 173)

Entre autores argentinos también se ha hecho referencia a estas transformaciones: 
"El sistema de mallas, nudos y redes jerárquicamente organizados, que constituye el sistema territorial, asegura el control sobre lo que puede ser distribuido, asignado, poseído dentro de determinado territorio" (Panaia, 2004, p.230)

Sin embargo, estas desigualdades ya no funcionan como en la época fordista. La organización territorial de ciertas actividades económicas se articula en espacios y economías en red, que replantean ciertas características de la relación entre lo local y lo global y junto con ello de las sociedades en su conjunto. En este aspecto, Sassen es quien mejor ha captado esta dualidad local/global:

“...estamos en presencia de geografías que hacen estallar los límites del contexto y la jerarquía tradicional de las escalas. Aunque se insertan en el contexto físico de los territorios nacionales, poco tienen que ver con el contexto que los rodea. En suma, estas nuevas geografías oscilan continuamente entre lo nacional y lo global, aunque es justo esta intersección lo que les otorga una mayor utilidad económica" (Sassen, 2010, pp.492-493)

Creemos que esta perspectiva, sin borrar las características y desigualdades propias de cada territorio, (re)significa y devuelve una cierta centralidad a los espacios locales. Lejos de querer homogeneizar procesos -que distan de serlo- sostenemos que es en la interacción donde se ponen en juego las capacidades de cada instancia global y local -expresado en sus actores e instituciones- para imponer sus demandas: en este sentido, el peso de los agentes económicos para imponer mayores condiciones aumentará en territorios con contrapesos estatales y sindicales más afines a sus intereses.

Respecto a la llegada de CCs, los servicios que se logran captar son los de baja jerarquización donde se obtiene competitividad vía costos laborales bajos y exenciones impositivas: no se observan estrategias que apuesten a ganar competitividad por arriba o competitividad "no costo" (Coriat, 1994) -con capacidad de especialización y de mayor calidad en productos y servicios-. Se mantiene así una división territorial del trabajo subordinada, donde el territorio y la colectividad que en él habita no obtiene mayores beneficios de la inserción de actividades globales en sus propias fronteras. En efecto, los territorios se conviertan en simple plataforma para las operaciones de CCs, sin lograr efectos multiplicadores de desarrollo: su localización en los distintos espacios locales no atrae la instalación de otras actividades, no inyecta grandes inversiones en inmuebles e infraestructura, no producen una mayor cualificación de sus recursos humanos, ni generan divisas para el territorio de localización; el efecto o externalidad casi exclusiva que genera es un mayor nivel de empleo no calificado y precario en el territorio; en concreto se logra una mayor retención de jóvenes empleados como operadores telefónicos en los principales centros urbanos del país.

En la localización de los CCs en espacios locales subordinados -como los aquí analizados- se podrían lograr ventajas en la captación de este tipo de actividades, como ser: a) obtener mayores márgenes de poder de negociación en la instalación de los CCs para lograr generar mayores vínculos de las empresas con la comunidad local; b) orientar recursos fiscales para recibir los segmentos de CCs de mayor valor agregado (tipo BPO) y c) intentar atraer la llegada del total o una parte parcialmente mayor del proceso productivo, más complejo en cuanto a calificaciones y tecnologías requeridas. 
El presente trabajo busca ser un aporte para el diseño de estrategias de desarrollo que contemplen las potencialidades del territorio. Conocer las características de la estructura de la actividad de CCs, su funcionamiento y las lógicas de su dinámica de expansión territorial sirve también para comprender que en ningún caso será eficaz una estrategia de desarrollo que se base aisladamente en la captación de este tipo de inversiones, desconectada de otras actividades tecnológicas que aporten mayor valor agregado.

No queremos caer en una mirada simplista: la interconectividad de la economía en el mundo global es una realidad que se profundiza cada vez más y es deseable que así sea. El desafío será obtener mejores condiciones de inserción global para los territorios, que comiencen a revertir las desigualdades observadas.

\section{BIBLIOGRAFÍA}

BARBETTI, P. El trabajo de los jóvenes en el conglomerado urbano Gran Resistencia: Desocupación y Precariedad laboral. Chaco: Centro de Estudios Sociales (CES) Universidad Nacional del Nordeste, 2003.

CASTELLS, M. La era de la información. Economía, Sociedad y Cultura. La Sociedad Red (vol.1). Bs. As.: Ed. Siglo XXI Editores Argentina, 2002.

CICCOLLELA, P. Reestructuración global, transformaciones económicas en la Argentina y reterritorialización de la región metropolitana de Buenos Aires. Hacia una ciudad competitiva, globalizada y excluyente. Revista de Estudios Regionales, Nro.43 pp 45-68, 1996.

CORIAT, B. Los desafios de la competitividad. Bs. As.: Eudeba, 1994.

DE MIRANDA OLIVEIRA JUNIOR, M. Brazilian Call Center Industry Report 2005. The Global Call center Industry Project, 2005.

DEL BONO, A. y HENRY, L. Tercerización de servicios en la Argentina: empleo y gestión de RRHH en los CC. Sistema de Información para la evaluación y el monitoreo del empleo, el trabajo y la inclusión social, Bs. As.: PNUD ARG/04/03, Ministerio de Trabajo, Empleo y Seguridad Social, 2009.

--------- La acción sindical en el marco de las formas globalizadas de producción. Reflexiones a partir de la expansión de los CC de servicios para exportación en Argentina. Revista Latinoamericana de Estudios del Trabajo Nro. 20, Segunda época, pp 7-31, 2008.

GARRO, S. Entre el hecho y el derecho: flexibilidad laboral y call centers en la Argentina postdevaluación. Ponencia IX Jornadas América Latina en el Nuevo Milenio: procesos, Crisis y Perspectivas en Facultad de Filosofia Universidad de Chile, 2008.

LASH, S. y URRY, J. Economías de signos y espacios. Londres: Amorrortu editores, 1994. 
LOPEZ, A., RAMOS, D. y STAROBINSKY, G. Clusters de software y servicios informáticos: los casos de Córdoba y Rosario a la luz de la experiencia internacional. Documentos de trabajo del CENIT, 2009.

MICHELI, J. Telemarketing y globalización en la economía mexicana. Memoria, Nro. 187, pag. 19-22, 2004.

PANAIA, M. Apuntes para la rediscusión del concepto de región en la Argentina actual. Revista de Estudios regionales, pp. 225-246, 2004.

SASSEN, S. Territorio, autoridad y derechos. De los ensamblajes medievales a los ensamblajes globales. Bs. As.: Katz Editores, 2010. 